\title{
Fatherless stem cells
}

Two independent research groups derive haploid mouse embryonic stem cells and propose their use for genetic screens.

The two copies of the mammalian autosomal genome, one inherited from the father and the other from the mother, are important for normal life, but they complicate genetic experiments. To study a recessive mutation implicated in disease, for instance, one must change both copies of the gene sequence. If one had haploid cells in hand, it would make life in the laboratory much easier.

This is what drove Ulrich Elling and Josef Penninger at the Institute of Molecular Biotechnology of the Austrian Academy of Sciences, Vienna, to revisit early attempts at deriving such cells. It had long been thought that the haploid state is incompatible with stable mammalian cells; surviving cells were typically seen to turn diploid. But, especially because Tijn Brummelkamp and colleagues had reported almost-haploid human tumor cells, Elling and Penninger thought it was worth looking into this problem again.

"If you look at some of [Nobel Prize laureate] Martin Evans' old papers," says Penninger, "they had haploid cells in there; the problem was they could not maintain them. Basically we did again what Martin did 28 years ago but we had a FACS sorter." The researchers used standard protocols to derive mouse embryos parthenogenetically (without fertilization), grew embryonic stem cells from these parthenotes and then used FACS to sort out the small remaining fraction of haploid cells. They found that, with periodic sorting, they could maintain haploid mouse embryonic stem cell lines (Elling et al., 2011).

Anton Wutz and Martin Leeb at the Wellcome Trust Center for Stem Cell Research, University of Cambridge, came at the problem from a different angle; they thought that haploid mouse cells might be a useful system in which to study the way cells count their X chromosomes. They were also influenced, explains Wutz, by the prevailing notion that normal haploid mammalian cells do not survive in culture and postulated that this might be because the cells mistakenly inactivate their single X chromosome. "We had a very nice theory before we actually did the experiment," Wutz laughs.

What the researchers instead saw, independently of Penninger and colleagues, was that many outgrowths from parthenogenetic mouse embryos in fact had a sizeable fraction of cells with only a single copy of the genome. Haploidy, in other words, is not as lethal to mammalian cells as it had previously been thought. The gentle conditions (so-called $2 \mathrm{i}$ conditions, in which two signaling pathways are inhibited) that Wutz and colleagues used to derive the embryonic stem cells may have contributed to the high percentage of haploid cells in their cultures (Leeb \& Wutz, 2011).

Both the Vienna and the Cambridge groups could mutagenize the haploid cells, the former with retroviral vectors and the latter with transposon-based vectors, and indicate that the cells will be useful for forward genetic screens. Both groups also note that, although the cells stay haploid for a few passages in culture, it is necessary to sort out the diploid cells that accumulate. What is more, differentiation results in 'diploidization' of the cells. This happens both in vitro, if the cells are differentiated in embryoid bodies, and in vivo, if the cells are used to generate chimeric mice. Critically, once they have diploidized, the cells are apparently broadly functional because they are capable of contributing to many tissue types in the mouse.

Although haploid cell biology is itself likely to be a rich research seam, the most immediate use of these cells will be in unbiased screens and other more targeted studies of gene function. In this context, the tendency of the cells to diploidize has important

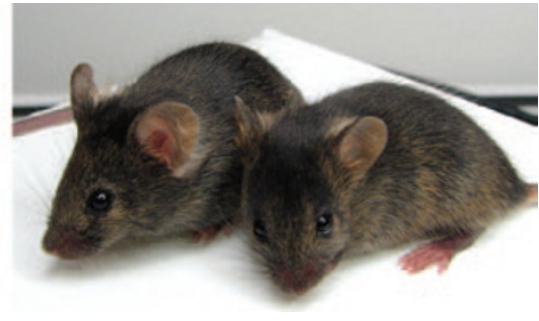

stem cells. Chromosome spread of a haploid mouse embryonic stem cell line and chimeric mice derived in part from the same cell line. Reproduced from Nature; image courtesy of Anton Wutz.

practical implications: it means that the cells can be mutagenized in the haploid state and then turned into homozygous diploids for phenotypic analysis. This has the advantage that the cells studied are more akin to normal mammalian cells, but the system is still an artificial one, as Penninger and Wutz both emphasize (cells could aquire genetic changes during diploidization, and paternally imprinted genes will not be expressed); experiments must therefore be designed accordingly. Penninger and colleagues, for instance, separate the effects of specific mutations from potential background effects by generating mutant alleles that are reversible with Cre recombinase.

Undoubtedly there are genome-wide resources of mutant haploid mouse embryonic stem cells on the horizon. In parallel with the efforts of the mouse gene-targeting consortia, and with cell collections for selection-free screening of homozygous mutant mouse embryonic stem cells (Horie et al., 2011), this will provide exciting possibilities for the cellular study of mammalian gene function.

\section{Natalie de Souza}

\section{RESEARCH PAPERS}

Elling, U. et al. Forward and reverse genetics through derivation of haploid mouse embryonic stem cells. Cell Stem Cell 9, 563-574 (2011).

Horie, K. et al. A homozygous mutant embryonic stem cell bank applicable for phenotype-driven genetic screening. Nat. Methods 8, 1071-1077 (2011).

Leeb, M. \& Wutz, A. Derivation of haploid embryonic stem cells from mouse embryos. Nature 479, 131-134 (2011). 